\title{
A case of subretinal neovascularization treated with intravitreal ranibizumab in a patient with idiopathic juxtafoveal retinal telangiectasis
}

\author{
Dimitrios Karagiannis' \\ Ilias Georgalas ${ }^{2}$ \\ loannis Ladas ${ }^{2}$ \\ Parikakis Eustratios' \\ Panagiotis Mitropoulos' \\ 'Second Department of \\ Ophthalmiatrion Eye Hospital ,NHS, \\ Athens, Greece; 'Department of \\ Ophthalmology, "G.Genimatas" \\ General Hospital, Athens, Greece
}

\begin{abstract}
A 65-year-old lady presented with decreased vision in her left eye (LE). Best corrected visual acuity (BCVA) was 1/20. Complete examination showed idiopathic juxtafoveal retinal telangiectasis associated with subretinal neovascularization and she was treated with intravitreal ranibizumab every month for three months in the LE. After four months, her BCVA increased to 3/10. Fluorescein angiography (FA) showed minimal leakage and optical coherence tomography (OCT) confirmed absence of intra- or subretinal fluid in the macula. Examinations were repeated monthly for another 12 months and showed no recurrence. Intravitreal ranibizumab showed promising results for subretinal neovascularization due to idiopathic juxtafoveal retinal telangiectasis. A prospective study with large series of patients and controls may be necessary in order to determine the effectiveness of this treatment.
\end{abstract}

Keywords: idiopathic juxtafoveal retinal telangiectasis, ranibizumab, subretinal neovascularization

\section{Introduction}

Gass and Blodi ${ }^{1}$ defined bilateral acquired retinal telangiectasis or perifoveal telangiectasia or type $2 \mathrm{~A}$ telangiectasis as a rare retinal disease characterized by retinal thickening temporal to the fovea, right-angle venules, retinal pigment epithelial (RPE) hyperplastic plaques, and crystalline deposits. Bilateral acquired retinal telangiectasis can be divided into five stages. The disease is usually becoming symptomatic through the sixth decade of life when complicated by subretinal neovascularization which is localized mostly subfoveally in stage 5 of the disease. ${ }^{1-3}$

We report the outcome of treatment with $0.5 \mathrm{mg}$ intravitreal ranibizumab in a patient with a subretinal neovascular membrane secondary to idiopathic juxtafoveal retinal telangiectasis.

\section{Case history}

A 65-year-old Caucasian lady presented to our clinic complaining of decreased vision in her left eye (LE). On presentation, best-corrected visual acuity (BCVA) was 10/10 in the right eye (RE) and 1/20 LE. Anterior segment examination was unremarkable in both eyes and intraocular pressures were within normal limits. Fundus biomicroscopy revealed in the right macula, slightly dilated right-angle veins with RPE hyperplasia beneath them (Figure 1A). In addition to these findings, in the left macula there was subretinal hemorrhage (Figure 1B). The clinical appearance of the macular area of the LE was consistent with idiopathic juxtafoveal retinal telangiectasis complicated by subretinal neovascularization. Fluorescein angiography (FA) and optical coherence tomography (OCT) were performed and confirmed the above diagnosis (Figures 1C-D, 2, 3A). OCT of the LE revealed the characteristic appearance of outer and inner retina having similar reflectivity and an area temporal to the fovea with high 


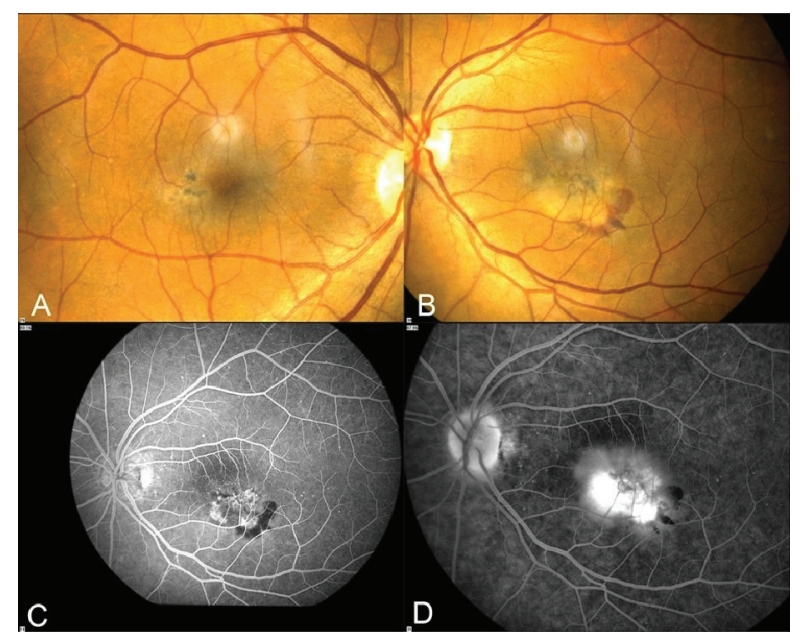

Figure IA) Right eye: Color fundus picture showing slightly dilated right-angle veins with retinal pigment epithelium hyperplasia beneath them. B) Left eye: Color fundus picture showing subretinal hemorrhage inferior and temporal to the fovea. C) Venous phase of FA of the LE depicts early hyperfluorescence corresponding to the neovascular membrane and hypofluorescence inferior to the membrane corresponding to the subretinal hemorrhage. D) Late phase of FA showing leakage from the neovascular membrane.

Abbreviations: FA, fluorescein angiography; LE, left eye; OCT, optical coherence tomography.

reflectivity corresponding to the temporal area of choroidal neovascularization (CNV) (Figure 3A), which was observed in the angiogram. After a discussion outlining the possible risks and benefits of the treatment, the patient was started on $0.5 \mathrm{mg}$ intravitreal ranibizumab in the LE. Based on the findings of FA and OCT which were performed monthly, the patient underwent three intravitreal injections of ranibizumab every month for three months. Following these injections, BCVA increased to $3 / 10$ and both clinical and angiographic appearances showed significant improvement with minimal leakage in FA and absence of intra- or subretinal fluid in OCT and significant reduction in retinal thickness; the RPE remained thickened (Figures 3B, 4A-B). Examinations were repeated monthly for another 12 months and showed no recurrence of CNV.

\section{Discussion}

Bilateral acquired retinal telangiectasis or retinal telangiectasis type 2A, according to Gass and Blodi, or type 2 perifoveal telangiectasia according to the classification by Yannuzzi is a symmetrical disease. ${ }^{1,3}$ The area involved is usually less than a disc diameter and is typically temporal to the fovea. ${ }^{1}$ Gas and Blodi divided the development of group $2 \mathrm{~A}$ telangiectasis into five stages. ${ }^{1}$ Stage 1 is found on FA on the asymptomatic fellow eye as staining at the level of the RPE. Stage 2 is characterized by the loss of transparency of the retina

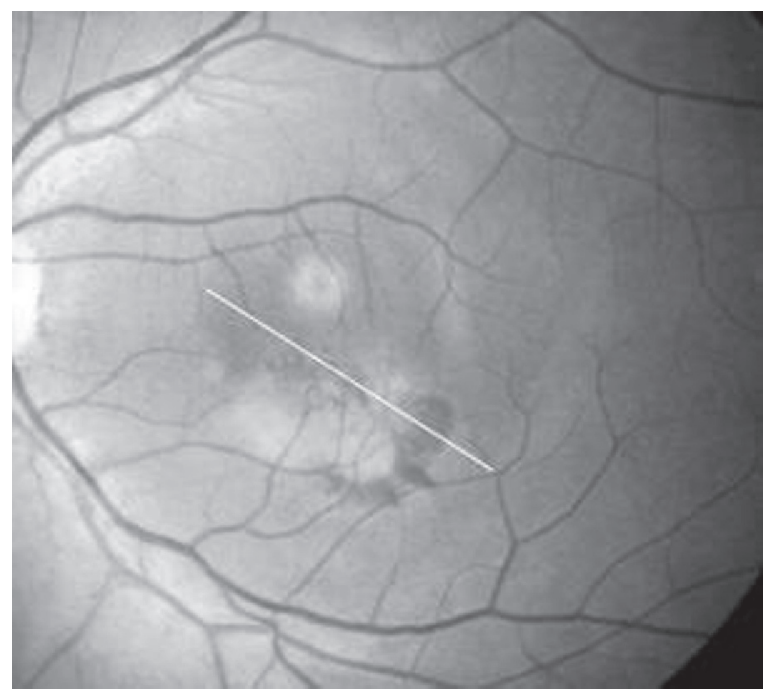

Figure 2 Oblique section of OCT of the LE.

Abbreviations: LE, left eye; OCT, optical coherence tomography.

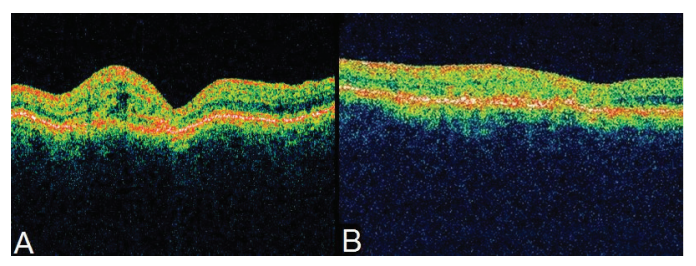

Figure 3A) Oblique section of OCT of the LE revealing the characteristic appearance of outer and inner retina having similar reflectivity and an area temporal to the fovea with high reflectivity corresponding to the temporal area of choroidal neovascularization observed in the angiogram. B) OCT of the same section of LE revealing significant reduction in retinal thickness. The RPE remains thickened.

Abbreviations: LE, left eye; OCT, optical coherence tomography; RPE, retinal pigment epithelium.

near the fovea and visible telangiectasis only in FA. In stage 3, FA shows capillary dilatation and staining. Stage 4 reveals migrating RPE cells appearing as stellate foci along the right-angle venules. Stage 5 , according to Gass and Blodi, or the proliferative stage according to

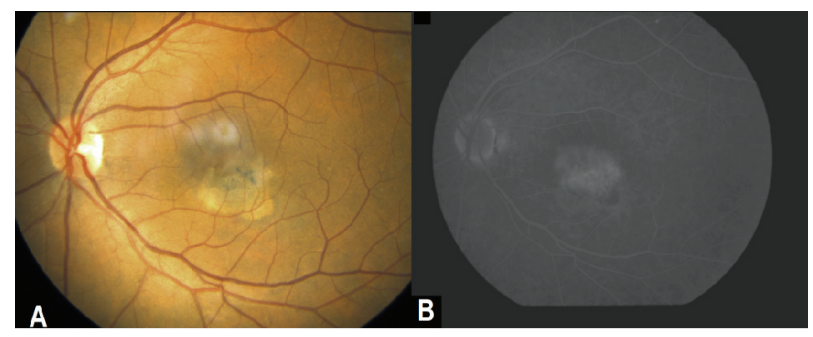

Figure 4 A) Left eye: Color fundus picture following ranibizumab treatment revealing significant improvement with no subretinal hemorrhage. B) late venous phase of FA of the LE showing minimal leakage due to telangiectasis but no signs of active neovascular membrane.

Abbreviations: FA, fluorescein angiography; LE, left eye. 
Table I Anti-VEGF for the treatment of subretinal neovascular membrane associated with type 2 idiopathic macular telangiectasia: Summarized review of the literature

\begin{tabular}{|c|c|c|c|c|c|}
\hline Author publication & Patients & Anti-VEGF & PDT & Follow-up (months) & Year of publication \\
\hline Rishi et al ${ }^{10}$ & 1 & Ranibizumab & Yes & 16 & 2008 \\
\hline Mandal et $\mathrm{al}^{7}$ & 6 & Bevacizumab & No & $3-6$ & 2008 \\
\hline Jorge et $\mathrm{al}^{8}$ & 1 & Bevacizumab & No & 24 & 2008 \\
\hline Ruys et $\mathrm{al}^{6}$ & 2 & Bevacizumab & Case I:Yes Case 2: No & $\mathrm{I}-3$ & 2007 \\
\hline Moon et $\mathrm{al}^{9}$ & I & Bevacizumab & No & 3 & 2007 \\
\hline
\end{tabular}

Abbreviations: PDT, photodynamic therapy; VEGF, vascular endothelial growth factor.

Yannuzzi's classification of the disease, is associated with subretinal neovascularization. ${ }^{1,3}$

According to Engelbrecht and colleagues ${ }^{2}$ the natural course of the disease in stage 5 is not good in $80 \%$ of cases, resulting to visual acuity of $1 / 20$ or less.

Snyers and colleagues showed that from four patients treated with photodynamic therapy (PDT), three of those retained their visual acuity, however, there was no significant improvement. ${ }^{4}$

Vascular endothelial growth factor (VEGF) is a major key player for angiogenesis in the eye in several physiologic and pathologic conditions. Concerning anti-VEGF therapy in ocular disease, clinical trials with ranibizumab show a beneficial effect for all subtypes of CNV including diseases such as pathological myopia and age-related macular degeneration. In addition, anti-VEGF therapy showed beneficial results, both clinically and anatomically, for idiopathic macular telangiectasia which was not associated with subretinal neovascularization. ${ }^{5}$

Recently, Ruys and colleagues have reported that the intravitreal delivery of bevacizumab (Avastin), with or without PDT was beneficial for both anatomical and visual outcome for subretinal neovascularization due to idiopathic juxtafoveal retinal telangiectasis. ${ }^{6}$ Other reports also support this beneficial outcome of bevacizumab (Avastin) (Table 1). ${ }^{7-9}$ In most of these cases the neovascularization ceased after two injections of bevacizumab. ${ }^{7}$

Also, combined PDT and intravitreal ranibizumab appears to be efficacious in the treatment of subretinal neovascularization associated with proliferative type 2 idiopathic macular telangiectasia (Table 1). ${ }^{10}$

In our case we chose to use ranibizumab since it has been designed for intraocular use; three intravitreal applications of ranibizumab, repeated monthly, alone without any additional session of PDT, were sufficient to cease the neovascularization during a follow-up time of 12 months.
In conclusion, intravitreal ranibizumab seems to have favorable results in the treatment of subretinal neovascularization due to idiopathic juxtafoveal retinal telangiectasis. Further studies are required to evaluate the above findings, although the implementation of large-series studies remains a challenge due to the rarity of cases with subretinal neovascularization secondary to idiopathic juxtafoveal retinal telangiectasis.

\section{Disclosure}

The authors report no conflicts of interest in this work.

\section{References}

1. Gass JD, Blodi BA. Idiopathic juxtafoveal retinal telangiectasis. Update of classification and follow-up study. Ophthalmology. 1993;100:1536-1546.

2. Engelbrecht NE, Aaberg TM Jr, Sung J, Lewis ML. Neovascular membranes associated with idiopathic juxtafoveolar telangiectasis. Arch Ophthalmol. 2002;120:320-324.

3. Yannuzzi LA, Bardal AM, Freund KB, Chen KJ, Eandi CM, Blodi B. idiopathic macular telangiectasia. Arch Ophthalmol. 2006;124:450-460.

4. Snyers B, Verougstraete C, Postelmans L, Leys A, Hykin P. Photodynamic therapy of subfoveal neovascular membrane in type $2 \mathrm{~A}$ idiopathic juxtafoveolar retinal telangiectasis. Am J Ophthalmol. 2004;137:812-819.

5. Charbel Issa P, Finger RP, Holz FG, Scholl HP. Eighteen-month follow-up of intravitreal bevacizumab in type 2idiopathic macular telangiectasia. Br J Ophthalmol. 2008;92:941-945.

6. Ruys J, De Laey JJ, Vanderhaeghen Y, Van Aken EH. Intravitreal bevacizumab (Avastin) for the treatment of bilateral acquired juxtafoveal retinal telangiectasis associated with choroidal neovascular membrane. Eye. 2007;21:1433-1434.

7. Mandal S, Venkatesh P, Abbas Z, Vohra R, Garg S. Intravitreal bevacizumab (Avastin) for subretinal neovascularization secondary to type 2A idiopathic juxtafoveal telangiectasia. Graefes Arch Clin Exp Ophthalmol. 2007;245:1825-1829.

8. Jorge R, Costa RA, Calucci D, Scott IU. Intravitreal bevacizumab (Avastin) associated with the regression of subretinal neovascularization in idiopathic juxtafoveolar retinal telangiectasis. Graefes Arch Clin Exp Ophthalmol. 2007;245:1045-1048.

9. Moon SJ, Berger AS, Tolentino MJ, Misch DM. Intravitreal bevacizumab for macular edema from idiopathic juxtafoveal retinal telaniectasis. Ophthalmic Surg Lasers Imaging. 2007;38:164-166.

10. Rishi P, Shroff D, Rishi E. Combined photodynamic therapy and intravitreal ranibizumab as primary treatment for subretinal neovascular membrane associated with type 2 idiopathic macular telangiectasia. Graefes Arch Clin Exp Ophthalmol. 2008;246:619-621. 
